Pacific

Journal of

Mathematics

COMBINATORIAL RIGIDITY IN CURVE COMPLEXES AND MAPPING CLASS GROUPS

KENNETH J. SHACKLETON

Volume $230 \quad$ No. 2

April 2007 


\title{
COMBINATORIAL RIGIDITY IN CURVE COMPLEXES AND MAPPING CLASS GROUPS
}

\author{
Kenneth J. ShaCKLETON
}

\begin{abstract}
In all possible cases, we prove that local embeddings between two curve complexes whose complexities do not increase from domain to codomain are induced by surface homeomorphism. This is our first main result. From this we can deduce our second, a strong local co-Hopfian property for mapping class groups.
\end{abstract}

\section{Introduction}

The curve complex $\mathscr{C}(\Sigma)$ associated to a surface $\Sigma$ was introduced by Harvey [1981] to encode the large scale geometry of Teichmüller space, and ultimately help decide the nonarithmeticity of the surface mapping class groups. It was later to play a central role in the proof by Minsky, Brock and Canary of Thurston's ending lamination conjecture [Brock et al. 2004].

We start by defining the curve complex, and throughout our surfaces will be compact, connected and orientable. We say that a simple loop on $\Sigma$ is trivial if it bounds a disc and peripheral if it bounds an annulus whose other boundary component belongs to $\partial \Sigma$. A curve on $\Sigma$ is a free homotopy class of a nontrivial and nonperipheral simple loop and we denote the set of these by $X(\Sigma)$. The intersection number of two curves $\alpha, \beta \in X(\Sigma)$, denoted $\iota(\alpha, \beta)$, is defined equal to $\min \{|a \cap b|: a \in \alpha, b \in \beta\}$. We say that two curves intersect minimally if they intersect once or they intersect twice with zero algebraic intersection and refer to either as the type of minimal intersection. We will later define the complexity of $\Sigma$, denoted $\kappa(\Sigma)$, as equal to the maximal number of distinct and disjoint curves that can be realised simultaneously.

When $\kappa(\Sigma) \geq 2$, the curve graph is the graph whose vertex set is $X(\Sigma)$ and we deem two distinct curves to span an edge if and only if they can be realised disjointly in $\Sigma$. When $\kappa(\Sigma)=1$, we say that two distinct curves are joined by an edge if and only if they intersect minimally. The curve complex associated to $\Sigma$ is the curve graph when $\kappa(\Sigma)=1$, making it isomorphic to a Farey graph, and the

MSC2000: primary 57M50; secondary 20F38.

Keywords: curve complex, mapping class group.

Supported by an EPSRC PhD scholarship. 
flag simplicial complex whose 1-skeleton is the curve graph when $\kappa(\Sigma) \geq 2$. In the latter case, $\mathscr{C}(\Sigma)$ has simplicial dimension precisely $\kappa(\Sigma)-1$.

For each curve $\alpha$ we denote by $X(\alpha)$ the set of all curves on $\Sigma$ distinct and disjoint from $\alpha$, that is the vertex set of the link of $\alpha$. This link is always connected whenever $\kappa(\Sigma)$ is at least three, and whenever $\kappa(\Sigma)$ is two any two elements of $X(\alpha)$ may be "chain-connected" by a finite sequence of curves in which any two consecutive curves have minimal intersection.

In this paper, we shall be discussing embeddings between two curve complexes whose complexities do not increase from domain to codomain and we shall find that these are all induced by surface homeomorphism, so long as we place a necessary but consistent hypothesis in one sporadic case. The argument we give is by an induction on complexity and requires little more than the connectivity of links in the curve complex over and above this. As such, our approach does not discriminate in terms of the topological type of a surface. Moreover, we actually only require the local injectivity of an embedding and we shall say more on this towards the end of this section.

All told, this generalises the automorphism theorem of Ivanov for surfaces of genus at least two, a proof of which is sketched in [Ivanov 1997, §2] and extended in [Korkmaz 1999] to all but the two-holed torus, and of Luo [2000], settled or proven in all cases. Making use of their combined result, Margalit [2004] establishes the analogue for automorphisms of another important surface complex called the pants complex. There are analogues for other surface complexes; see [Schmutz Schaller 2000] as one example.

Theorem 1. Suppose that $\Sigma_{1}$ and $\Sigma_{2}$ are two compact and orientable surfaces of positive complexity such that the complexity of $\Sigma_{1}$ is at least that of $\Sigma_{2}$, and that when they have equal complexity at most three they are homeomorphic or one is the three-holed torus. Then, any simplicial embedding from $\mathscr{C}\left(\Sigma_{1}\right)$ to $\mathscr{C}\left(\Sigma_{2}\right)$ (preserving the separating type of each curve when the two surfaces are homeomorphic to the two-holed torus) is induced by a surface homeomorphism.

This covers all possibilities. We remind ourselves that there exist isomorphisms between the curve complex of the closed surface of genus two and the six-holed sphere, the two-holed torus and the five-holed sphere and finally the one-holed torus and the four-holed sphere and that there exists an automorphism of the curve complex associated to the two-holed torus that sends a nonseparating curve to an outer curve (see [Luo 2000] for more details). These are examples of embeddings not induced by a surface homeomorphism. Finally, we point out that there exist embeddings on curve complexes with complexity increasing from domain to codomain not induced by a surface embedding: Easy examples are provided by taking some proper subsurface $\Sigma_{1}$ of $\Sigma_{2}$, and modifying the induced embedding 
on curve complexes by instead taking just one curve on $\Sigma_{1}$ to a curve on $\Sigma_{2}$ outside of $\Sigma_{1}$.

Among other things, Theorem 1 completes one study of a particular class of selfembedding, initiated by Irmak. This class comprises the superinjective maps, and by definition each preserves the nonzero intersection property of a pair of curves. Irmak [2004b] first showed that a superinjective self-map is induced by a surface homeomorphism provided the surface is closed and of genus at least three, then [2006] extended this result to nonclosed surfaces of genus at least three and surfaces of genus two with at least two holes, and to the remaining two types of genus two surface [2004a]. Following a now standard strategy, first set out by Ivanov, this holds consequences for the mapping class groups of the corresponding surfaces.

The mapping class group Map $\Sigma$ is the group of all self-homeomorphisms of the surface $\Sigma$, up to homotopy. This is sometimes known as the extended mapping class group, for it contains the group of orientation preserving mapping classes as an index two subgroup. Some of its other subgroups, in particular the Johnson kernel and the Torelli group, are of wide interest; see, respectively, [Brendle and Margalit 2004] and [Farb and Ivanov 2005], and references contained therein.

The mapping class group has a natural simplicial action on the curve complex, determined by first lifting a curve to a representative loop and then taking the free homotopy class of the image under a representative homeomorphism. The kernel of this action, $\operatorname{Ker} \Sigma$, is almost always trivial: the only exceptions lie in low complexity, where this kernel is isomorphic to $\mathbb{Z}_{2}$ and generated by the hyperelliptic involution when $\Sigma$ is the one-holed torus, the two-holed torus, or the closed surface of genus two or isomorphic to $\mathbb{Z}_{2} \oplus \mathbb{Z}_{2}$ and generated by two hyperelliptic involutions when the four-holed sphere; this was proved in [Birman 1974; Viro 1972]. For a detailed account of the mapping class group and its subgroups, see [Ivanov 1992] as one place to start.

Theorem 1 implies the following strong co-Hopfian property for mapping class groups. Among other things Theorem 2 has some familiar consequences, namely it follows that the commensurator group of a mapping class group is isomorphic to the same mapping class group and that the outer automorphism group of a mapping class group is trivial. It also follows that mapping class groups do not admit a faithful action on another curve complex of no greater dimension.

Theorem 2. Suppose that $\Sigma_{1}$ and $\Sigma_{2}$ are two compact and orientable surfaces such that the complexity of $\Sigma_{1}$ is at least that of $\Sigma_{2}$ and at least two, and that whenever they both have complexity equal to three they are homeomorphic, though not to the closed surface of genus two, or one is the three-holed torus and that when they both have complexity two they are homeomorphic to the five-holed sphere. Suppose that $H$ is a finite index subgroup of the mapping class group Map $\Sigma_{1}$. Then, every injection of $H$ into Map $\Sigma_{2}$ is the restriction of an inner automorphism of Map $\Sigma_{1}$. 
The existence of such a homomorphism is understood to imply the two surfaces are equal. Theorem 2 is a generalisation of [Ivanov and McCarthy 1999, Theorem 4], which deals with injections defined on mapping class groups associated to surfaces of positive genus.

The combined superinjectivity theorem implies Theorem 2 when the two surfaces under consideration are homeomorphic and have genus at least three, or genus at least two and one hole, and Irmak [2004a] describes a noninner automorphism for the closed surface of genus two. Bell and Margalit [2005] extend this to spheres with at least five holes, and Behrstock and Margalit [2006] to genus one surfaces with at least three holes in addition to finding a commensurator for the mapping class group of the two-holed torus not induced by an inner automorphism. The remaining cases, namely the mapping class group of the four-holed sphere and of the one-holed torus, also have noninner injections on finite index subgroups, as both are virtually free groups. We remark the braid groups on at least four strands, modulo centre have the co-Hopfian property; see [Bell and Margalit 2006].

The general approach we need for Theorem 2 follows that given by Ivanov, translating an injection on a finite index subgroup to an embedding on curve complexes. This is now a well-established strategy on which we have nothing to add, and a thorough account can be found in both [Bell and Margalit 2005] and [Irmak 2004b].

Though all our arguments are phrased in terms of embeddings, they only ever need the simplicial and local injectivity properties of such maps. We can therefore record the following generalisation of Theorem 1, the first of two main results. Recall that a star is the union of all edges incident on a common vertex.

Theorem 3. Suppose that $\Sigma_{1}$ and $\Sigma_{2}$ are two compact and orientable surfaces of positive complexity such that the complexity of $\Sigma_{1}$ is at least that of $\Sigma_{2}$, and that when they have equal complexity at most three they are homeomorphic or one is the three-holed torus. Then, any simplicial map from $\mathscr{b}\left(\Sigma_{1}\right)$ to $\mathscr{C}\left(\Sigma_{2}\right)$ injective on every star (and preserving the separating type of each curve when both surfaces are homeomorphic to the two-holed torus) is induced by a surface homeomorphism.

Again, this covers all possibilities. We remark that proving a local embedding is induced by a surface homeomorphism would appear the most direct way of seeing that it must also be a global embedding. Furthermore, we conjecture that the pants complex also exhibits such local-to-global rigidity.

From Theorem 3 we can deduce, using a careful application of Ivanov's strategy, the following local version of Theorem 2. This is one interpretation of local injectivity for mapping class groups, and a proof is completed in [Shackleton 2005, §3.3]. Among other things, it follows that a self-homomorphism of a mapping class group injective on every curve stabiliser is the restriction of an inner automorphism. 
Theorem 4. Suppose that $\Sigma_{1}$ and $\Sigma_{2}$ are two compact and orientable surfaces such that the complexity of $\Sigma_{1}$ is at least that of $\Sigma_{2}$ and at least two, and that whenever they both have complexity equal to three they are homeomorphic, though not to the closed surface of genus two, or one is the three-holed torus and that whenever they both have complexity two they are homeomorphic to the five-holed sphere. Suppose that $H$ is a finite index subgroup of the mapping class group Map $\Sigma_{1}$. Then, every homomorphism of $H$ into Map $\Sigma_{2}$ injective on every curve stabiliser in $H$ is the restriction of an inner automorphism of Map $\Sigma_{1}$.

Note once more that the existence of such a homomorphism is understood to imply the two surfaces are equal.

Investigations into arbitrary homomorphisms from a mapping class group associated to a closed surface of genus at least one to another mapping class group associated to a closed surface of smaller genus have been made Harvey and Korkmaz [2005], who found that every such homomorphism has finite image. Their approach seems to make essential use of the existence of torsion in mapping class groups and, as mapping class groups are virtually torsion free, it would be of some interest to find a way around this so as to consider finite index subgroups.

\section{Embeddings between curve complexes}

For any compact, connected and orientable surface $\Sigma$ the complexity $\kappa(\Sigma)$ of $\Sigma$ is defined to be equal to 3genus $\Sigma+|\partial \Sigma|-3$. This is perhaps nonstandard, since complexity is often taken to be equal to the simplicial dimension of the curve complex, but the additivity of $\kappa$ best suits our induction argument. By way of example, the one-holed torus and the four-holed sphere are the only surfaces of complexity one, the two-holed torus and the five-holed sphere are the only surfaces of complexity two, and the closed surface of genus two, the three-holed torus and the six-holed sphere are the only surfaces of complexity three. On occasion we refer to these as the low complexity surfaces.

We shall abuse notation slightly by viewing each curve as a vertex, as a class of loops, or as a simple loop already realised on $\Sigma$. Our interpretation will be apparent from the context. We say that a curve is separating if its complement is not connected, and otherwise say it is nonseparating. We say that a curve is an outer curve if it is separating and if it bounds a two-holed disc (equivalently, a threeholed sphere). These are usually known as boundary curves in the literature, but here we need to avoid confusing them with the components of $\partial \Sigma$. A multicurve on $\Sigma$ is a collection of distinct and disjoint curves, and a pants decomposition of $\Sigma$ is a maximal multicurve. A pair of pants in $\Sigma$ is an essential subsurface homeomorphic to a compact three-holed sphere. 
We say that two curves in a pants decomposition $P$ are adjacent in $P$ if they appear in the boundary of a three-holed sphere complementary to $P$. This is slightly unfortunate terminology that seems to be a long way to becoming standard; we sincerely hope that any confusion between adjacency in the curve complex and adjacency in a pants decomposition will be obviated by the context.

The structure of our argument is broadly as follows. We establish a short list of topological properties verified by any embedding on curve complexes from which we easily deduce, among other things, that the existence of such an embedding implies the two surfaces have equal complexity and then, with more work, almost always means the two surfaces under consideration are homeomorphic. For the time being, we refer to embeddings between two apparently distinct curve complexes as cross-embeddings. Dealing with embeddings in low complexity typically requires individual arguments and it therefore streamlines our work if we do this separately, as we do in Lemma 13. The proof of Theorem 1 is then completed by an induction on complexity, where we cut the surface along a curve. As embeddings behave well on the topological type of a curve, the resulting surfaces are again homeomorphic. For the induction argument to pass through complexity one (sub)surfaces, we will need to know that embeddings preserve minimal intersection.

We start by showing, in turn, that embeddings send pants decompositions to pants decompositions, they preserve a form of small intersection and they preserve adjacency and nonadjacency in a pants decomposition.

Lemma 5. Suppose that $\Sigma_{1}$ and $\Sigma_{2}$ are two compact and orientable surfaces such that the complexity of $\Sigma_{1}$ is at least that of $\Sigma_{2}$. Then, any embedding $\phi$ from $\mathscr{C}\left(\Sigma_{1}\right)$ to $\mathscr{C}\left(\Sigma_{2}\right)$ sends pants decompositions to pants decompositions.

Proof. This follows for complexity reasons and because $\phi$ is simplicial and injective.

To make sense of the following lemma, we must define what we mean by the subsurface of $\Sigma$ filled by two curves $\alpha$ and $\beta$. Letting $N(\alpha \cup \beta)$ denote a closed regular neighbourhood of $\alpha \cup \beta$ in $\Sigma$, we augment $N(\alpha \cup \beta)$ by taking its union with all the complementary discs whose boundary is contained in $N(\alpha \cup \beta)$ and all the complementary annuli with one boundary component in $\partial \Sigma$ and the other in $N(\alpha \cup \beta)$. The resulting subsurface of $\Sigma$ is well defined up to isotopy and is what we mean by the subsurface filled by $\alpha$ and $\beta$. Whenever a third curve enters the subsurface filled by two curves, it must intersect at least one of these two curves.

Lemma 6. Suppose that $\Sigma_{1}$ and $\Sigma_{2}$ are two compact and orientable surfaces such that the complexity of $\Sigma_{1}$ is at least that of $\Sigma_{2}$. Let $\phi$ be any embedding from $\mathscr{C}\left(\Sigma_{1}\right)$ to $\mathscr{C}\left(\Sigma_{2}\right)$ and let $\alpha, \beta$ be any two curves in $\Sigma_{1}$ that fill either a four-holed sphere or a one-holed torus. Then, $\phi(\alpha)$ and $\phi(\beta)$ fill either a four-holed sphere or a one-holed torus in $\Sigma_{2}$. 
Proof. Let $Q$ be any maximal multicurve in $\Sigma_{1}$ such that each curve is disjoint from both $\alpha$ and $\beta$. For complexity reasons, $\phi(Q)$ is a maximal multicurve disjoint from both $\phi(\alpha)$ and $\phi(\beta)$. In particular, as $\phi$ is injective and simplicial so $\phi(\alpha)$ and $\phi(\beta)$ must together fill either a four-holed sphere or a one-holed torus.

We say that two curves have small intersection if they together fill either a fourholed sphere or a one-holed torus, and refer to either as the type of the small intersection. Any two curves that intersect minimally also have small intersection, but the converse does not hold.

Lemma 7. Suppose that $\Sigma_{1}$ and $\Sigma_{2}$ are two compact and orientable surfaces such that the complexity of $\Sigma_{1}$ is at least that of $\Sigma_{2}$. Let $P$ be any pants decomposition of $\Sigma_{1}$ and let $\phi$ be any embedding from $\mathscr{C}\left(\Sigma_{1}\right)$ to $\mathscr{C}\left(\Sigma_{2}\right)$. Then, any two curves adjacent in $P$ are sent by $\phi$ to two curves adjacent in $\phi(P)$ and any two curves in $P$ that are not adjacent in $P$ are sent by $\phi$ to two curves not adjacent in $\phi(P)$.

Proof. The first part follows from Lemma 6: For any two curves $\alpha_{1}$ and $\alpha_{2}$ adjacent in $P$, there exists a curve $\delta$ having small intersection with both and disjoint from every other curve in $P$. This is preserved under $\phi$ and so $\phi\left(\alpha_{1}\right)$ and $\phi\left(\alpha_{2}\right)$ are adjacent in $\phi(P)$.

Similarly, if two curves $\alpha_{1}, \alpha_{2}$ are not adjacent in $P$ we can find two disjoint curves $\delta_{1}, \delta_{2}$ such that $\delta_{1}$ has small intersection with $\alpha_{1}$ but is disjoint from $\alpha_{2}$ and $\delta_{2}$ has small intersection with $\alpha_{2}$ but is disjoint from $\alpha_{1}$ and both $\delta_{1}, \delta_{2}$ are disjoint from every other curve in $P$. If $\phi\left(\alpha_{1}\right)$ and $\phi\left(\alpha_{2}\right)$ are adjacent in $\phi(P)$ then $\phi\left(\delta_{1}\right)$ and $\phi\left(\delta_{2}\right)$ must intersect. As $\phi$ is simplicial, this is a contradiction.

The import of Lemma 5, Lemma 6 and Lemma 7 is perhaps best understood by associating to a pants decomposition $P$ a certain graph. The vertices of this graph are the curves in $P$, and any two distinct vertices span an edge if and only if they correspond to adjacent curves in $P$. Lemma 7 not only tells us that any embedding $\phi$ induces a map between adjacency graphs, but that this map is actually an isomorphism. Cut points in the graph correspond to nonouter separating curves, and noncut points correspond to outer or nonseparating curves.

This graph, and the ideas bound by Lemma 7, were independently and simultaneously discovered by Behrstock and Margalit. Their approach, published in a joint paper [2006], and the arguments they give will deal with all superinjective maps for two homeomorphic surfaces of complexity at least three. From this they also deduce that the commensurator group of a mapping class group is isomorphic to the same mapping class group. We both refer to such a graph as an adjacency graph.

We can just as well speak of an adjacency graph associated to a multicurve $Q$, in which the vertices again correspond to the curves in $Q$ and any two vertices are declared adjacent if their corresponding curves border a common pair of pants in 


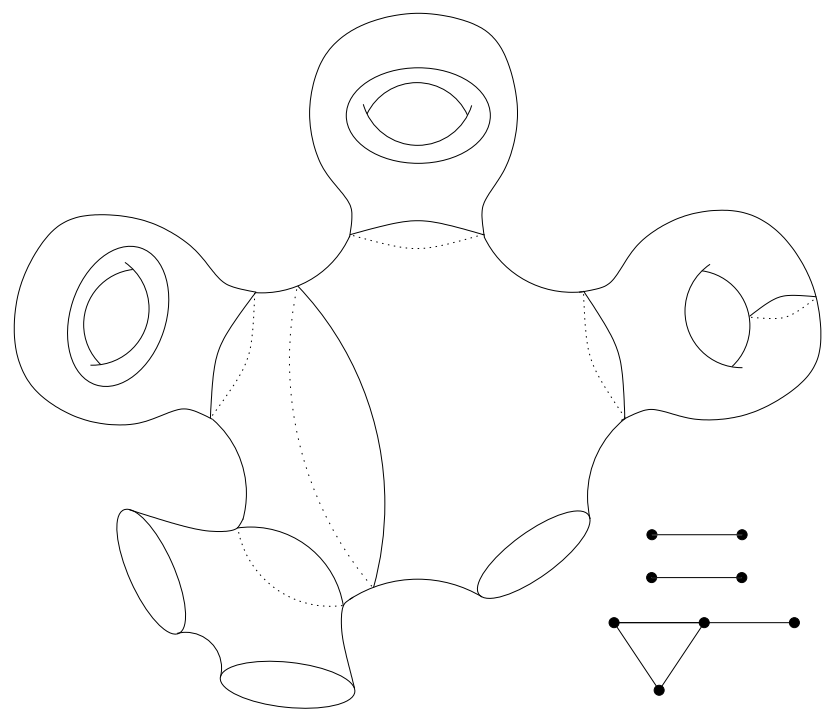

Figure 1. A codimension 1 multicurve, with its adjacency graph.

the surface complement of $Q$. There is a subtle point to be made here, namely that the complementary graph of a vertex in a pants adjacency graph will not in general be the adjacency graph of the multicurve that results by removing the corresponding curve from the pants decomposition. It will however be the adjacency graph that results from cutting the surface along this curve. By way of example, on removing a curve $\alpha$ from a pants decomposition $P$ any curves that together bound a complementary four-holed sphere will not necessarily be adjacent in the adjacency graph of $P-\{\alpha\}$. (See Figure 1 for an example.) This observation will be important later when we come to look at outer curves. It does however hold that a curve complex embedding induces an isomorphism between multicurve adjacency graphs.

Lemma 8. Suppose that $\Sigma_{1}$ and $\Sigma_{2}$ are compact and orientable surfaces such that the complexity of $\Sigma_{1}$ is at least that of $\Sigma_{2}$. Let $Q$ be any multicurve of $\Sigma_{1}$ and let $\phi$ be any embedding from $\mathscr{C}\left(\Sigma_{1}\right)$ to $\mathscr{C}\left(\Sigma_{2}\right)$. Then, $\phi$ induces an isomorphism from the adjacency graph of $Q$ to the adjacency graph of $\phi(Q)$.

Proof. We make use of Lemma 7. Extend $Q$ to a pants decomposition $P$ of $\Sigma_{1}$. If two curves are adjacent in $Q$ then they either border a pair of pants with a third curve from $Q$ or they border a pair of pants meeting $\partial \Sigma$. This remains so in $P$, and is preserved on applying $\phi$. To show nonadjacency is preserved, consider any two curves not adjacent in $Q$ and arrange for them to be nonadjacent in $P$. This is preserved under $\phi$. 
As embeddings between curve complexes induce isomorphisms on adjacency graphs and graph isomorphisms send cut points to cut points, so embeddings must send nonouter separating curves to nonouter separating curves.

Lemma 9. Suppose that $\Sigma_{1}$ and $\Sigma_{2}$ are two compact and orientable surfaces such that the complexity of $\Sigma_{1}$ is at least that of $\Sigma_{2}$. Then, any embedding $\phi$ from $\mathscr{C}\left(\Sigma_{1}\right)$ to $\mathscr{C}\left(\Sigma_{2}\right)$ sends nonouter separating curves to nonouter separating curves.

We use the adjacency graph to distinguish between nonseparating and outer curves.

Lemma 10. Suppose $\Sigma_{1}$ and $\Sigma_{2}$ are two compact and orientable surfaces such that the complexity of $\Sigma_{1}$ is at least that of $\Sigma_{2}$ and that whenever they have equal complexity at most three they are homeomorphic and not the two-holed torus. Let $\phi: \mathscr{C}\left(\Sigma_{1}\right) \rightarrow \mathscr{C}\left(\Sigma_{2}\right)$ be any embedding. Then, $\phi$ takes nonseparating curves to nonseparating curves.

Proof. We note that the $\phi$-image of a nonseparating curve can never be a nonouter separating curve, for otherwise we see a noncut point sent to a cut point in some pants adjacency graph. Suppose that $\alpha$ is a nonseparating curve in $\Sigma_{1}$. When $\kappa\left(\Sigma_{1}\right)$ is at least four we can find a pants decomposition $P$ extending $\alpha$ in which $\alpha$ corresponds to a vertex in the adjacency graph of $P$ of valence three or four. As $\phi$ induces an isomorphism on the adjacency graph, so $\phi(\alpha)$ must have the same valence. As outer curves only ever correspond to vertices of valence at most two, so $\phi(\alpha)$ can only be nonseparating.

With the exception of the two-holed torus, all cases in which $\Sigma_{1}$ has complexity at most two hold since there is only ever one type of curve. In complexity three, when $\Sigma_{1}$ is the six-holed sphere our claim holds vacuously and when $\Sigma_{1}$ is the closed surface of genus two our claim follows from Lemma 9 by noting that every pants decomposition contains at most one separating curve.

The only nontrivial case in low complexity is that of $\Sigma_{1}$ and $\Sigma_{2}$ both homeomorphic to the three-holed torus. In which case, there are only two pants adjacency graphs, up to isomorphism, but three different pants decompositions, up to the action of the mapping class group. For this reason, we need to argue differently: If there is a nonseparating curve sent by $\phi$ to an outer curve, then there is an outer curve $\alpha$ sent by $\phi$ to a nonseparating curve. To see this, extend this nonseparating curve to a pants decomposition containing a nonouter separating curve. By appealing to Lemma 9, we see that the third curve in this pants decomposition will suffice. Now extend $\alpha$ to a second pants decomposition containing two nonseparating curves $\delta_{1}$ and $\delta_{2}$. The $\phi$-image of at least one of these, say $\delta_{1}$, is again a nonseparating curve. Choose any two disjoint curves $\gamma_{1}, \gamma_{2}$ in $\Sigma_{1}$ that have small intersection with $\delta_{1}$ and $\alpha$ but disjoint from $\alpha$ and $\delta_{1}$, respectively. Now $\phi\left(\delta_{1}\right)$ and $\phi(\alpha)$ border a common pair of pants in $\Sigma_{2}$ invaded by $\phi\left(\gamma_{1}\right)$ and $\phi\left(\gamma_{2}\right)$. We see that 


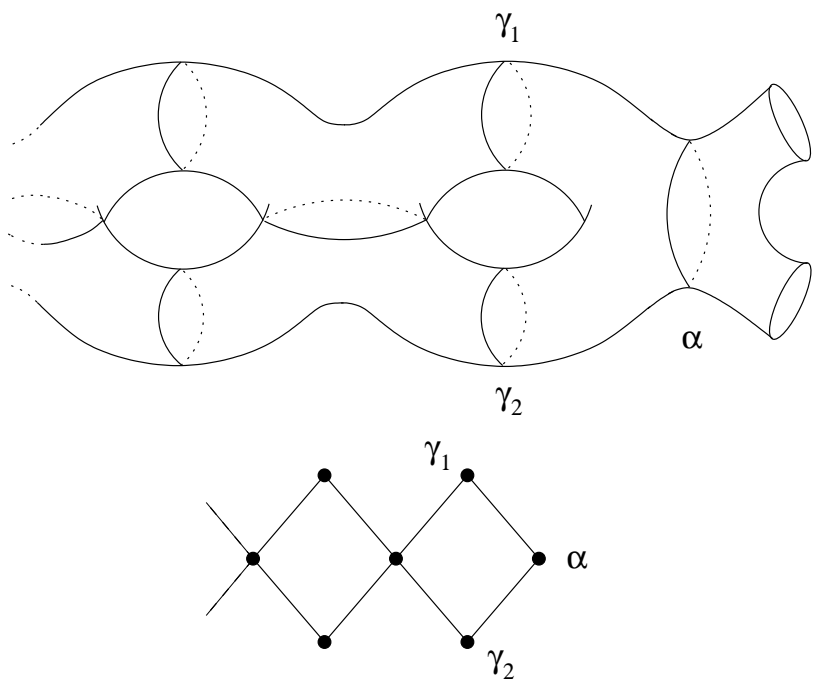

Figure 2. A convenient extension of $\alpha$ to a pants decomposition.

the $\phi$-images of both $\gamma_{1}$ and $\gamma_{2}$ are forced to intersect, and this is a contradiction.

Lemma 11. Suppose that $\Sigma_{1}$ and $\Sigma_{2}$ are two compact and orientable surfaces such that the complexity of $\Sigma_{1}$ is at least that of $\Sigma_{2}$, and that whenever they have equal complexity at most three they are homeomorphic and not the two-holed torus. Then, any embedding $\phi$ from $\mathscr{C}\left(\Sigma_{1}\right)$ to $\mathscr{C}\left(\Sigma_{2}\right)$ sends outer curves to outer curves.

Proof. We note that this holds vacuously when $\left|\partial \Sigma_{1}\right|$ is at most one. In any case, let us suppose for contradiction that $\alpha$ is an outer curve in $\Sigma_{1}$ sent by $\phi$ to a nonouter curve in $\Sigma_{2}$. We note that $\phi(\alpha)$ can not be a separating curve, for $\alpha$ can never correspond to a cut point in a pants adjacency graph, and so $\phi(\alpha)$ must be a nonseparating curve. If $\kappa\left(\Sigma_{1}\right)$ is at least four then we can extend $\alpha$ to a pants decomposition $P$ in which the two curves adjacent to $\alpha$, denoted $\gamma_{1}$ and $\gamma_{2}$, are not adjacent in the adjacency graph of $P-\{\alpha\}$. As $\alpha$ is an outer curve, we note that $\gamma_{1}$ and $\gamma_{2}$ are adjacent in $P$. According to Lemma 8, $\phi\left(\gamma_{1}\right)$ and $\phi\left(\gamma_{2}\right)$ can only, together with $\partial \Sigma_{2}$, border a four-holed sphere containing $\phi(\alpha)$. However, by assumption $\phi(\alpha)$ is not an outer curve. Therefore, $\phi\left(\gamma_{1}\right)$ and $\phi\left(\gamma_{2}\right)$ are not adjacent in $\phi(P)$ and this is contrary to the statement of Lemma 7. (See Figure 2 for one example.)

Once more, the only remaining nontrivial case in low complexity is that of the three-holed torus. Suppose that $\alpha$ is an outer curve sent to a nonseparating curve by $\phi$. Extend $\alpha$ to a pants decomposition $P$ containing a separating curve. Then 
the nonseparating curve in $P$ is sent to an outer curve by $\phi$, and this is contrary to Lemma 10.

It now follows that small subsurfaces can not change topological type under embeddings.

Lemma 12. Suppose that $\Sigma_{1}$ and $\Sigma_{2}$ are two compact and orientable surfaces such that the complexity of $\Sigma_{1}$ is at least that of $\Sigma_{2}$ and that when both have equal complexity at most three they are homeomorphic and not the two-holed torus. Let $Z$ be any essential subsurface of $\Sigma_{1}$ of complexity one and bordered by a single curve $\beta$. Then, for any embedding $\phi$ from $\mathscr{C}\left(\Sigma_{1}\right)$ to $\mathscr{C}\left(\Sigma_{2}\right)$, the essential subsurface $\phi(Z)$ of $\Sigma_{2}$ filled by $\phi(X(Z))$ is homeomorphic to $Z$.

Proof. Such a change in topology would otherwise force $\phi$ to send a nonseparating curve to an outer curve or an outer curve to a nonseparating, contrary to Lemmas 10 and 11 , respectively.

We can finally rule out cross-embeddings, and thereafter we regard the two surfaces as being equal and denote both by $\Sigma$.

Lemma 13. Suppose that $\Sigma_{1}$ and $\Sigma_{2}$ are two compact and orientable surfaces such that the complexity of $\Sigma_{1}$ is at least that of $\Sigma_{2}$, and that whenever they have complexity at most two they are homeomorphic and whenever they have complexity equal to three they are either homeomorphic or one is the three-holed torus. Then, there is an embedding $\phi: \mathscr{C}\left(\Sigma_{1}\right) \rightarrow \mathscr{C}\left(\Sigma_{2}\right)$ only if $\Sigma_{1}$ and $\Sigma_{2}$ are homeomorphic.

Proof. The existence of such an embedding implies the complexities $\kappa\left(\Sigma_{1}\right)$ and $\kappa\left(\Sigma_{2}\right)$ are equal. When $\kappa\left(\Sigma_{1}\right)$ is at least four, we know that any such embedding must send separating curves to separating curves. We recall that the size of a maximal collection of distinct and disjoint separating curves in $\Sigma_{1}$ is precisely 2 genus $\left(\Sigma_{1}\right)+\left|\partial \Sigma_{1}\right|-3$. By our earlier work, this is at most 2 genus $\left(\Sigma_{2}\right)+\left|\partial \Sigma_{2}\right|-3$ and so $\operatorname{genus}\left(\Sigma_{1}\right) \geq \operatorname{genus}\left(\Sigma_{2}\right)$.

To prove equality, we take $Q$ to be a maximal collection of distinct and disjoint curves on $\Sigma_{1}$ each bounding a one-holed torus. That is, $Q$ has genus $\left(\Sigma_{1}\right)$ curves. According to Lemma 12, each curve in $\phi(Q)$ must also bound a one-holed torus in $\Sigma_{2}$. We deduce genus $\left(\Sigma_{1}\right) \leq$ genus $\left(\Sigma_{2}\right)$. Combining the two inequalities we have genus $\left(\Sigma_{1}\right)=\operatorname{genus}\left(\Sigma_{2}\right)$, and $\Sigma_{1}$ and $\Sigma_{2}$ are homeomorphic.

Turning to the low complexity surfaces, there are no embeddings from the curve complex of the six-holed sphere or closed surface of genus two to that of the threeholed torus. To see this, extend an outer or nonseparating curve $\alpha$ in $\Sigma_{1}$ to a pants decomposition $P$ consisting only of outer or nonseparating curves, respectively, and choose a separating curve $\beta$ disjoint from both curves in $P-\{\alpha\}$ and therefore of small intersection with $\alpha$. We may assume that if any curve in $\phi(P)$ is outer 
then it is $\phi(\alpha)$. Now $\phi(\beta)$ is a nonouter separating curve intersecting $\phi(\alpha)$ and it follows that $\phi(\beta)$ must intersect another curve in $\phi(P)$. This is a contradiction.

The remaining cases, namely from the curve complex of the three-holed torus to the curve complex of the six-holed sphere or of the closed surface of genus two, are covered as follows: For any pants decomposition $P$ in $\Sigma_{1}$ comprising only of nonseparating curves, choose a nonouter separating curve $\beta$ meeting only two curves in $P$. When $\Sigma_{2}$ is the six-holed sphere, according to Lemma 8 each curve in $P$ can only go to an outer curve. By Lemma 6 , small intersection is preserved. Now any nonouter separating curve in the six-holed sphere meets either only one curve or all three curves in a pants decomposition made up entirely of outer curves. It follows that $\phi(\beta)$ meets every curve in $\phi(P)$, and this is a contradiction. This simultaneously deals with $\Sigma_{2}$ the closed surface of genus two.

To allow the induction argument to pass through complexity one surfaces unhindered, we need the following lemma on minimal intersection in those subsurfaces bordered by a single curve. This relies on what is a well-established argument, given in [Ivanov 1997] for intersection one and in [Korkmaz 1999; Luo 2000] for intersection two with zero algebraic intersection. Although both are stated for automorphisms, both apply in our setting.

Lemma 14. Suppose that $\Sigma$ is a compact and orientable surface of positive complexity and not homeomorphic to the two-holed torus. Suppose that $Z$ is an essential subsurface of $\Sigma$ of complexity one and bordered by a single curve $\beta$. Then, any embedding $\phi: \mathscr{C}(\Sigma) \rightarrow \mathscr{C}(\Sigma)$ preserves minimal intersection and its type on $X(Z)$.

This closes our study of the topological properties of curve complex embeddings, and the promised induction argument now starts with a look at the Farey graph.

Lemma 15. Every simplicial embedding from a Farey graph F F to itself is an automorphism.

Proof. We note that each edge in $\mathscr{F}$ separates and belongs to exactly two 3-cycles and that such a map sends 3 -cycles to 3 -cycles. Thus, any embedding $\phi$ on $\mathscr{F}$ induces an embedding $\phi^{*}$ on the dual graph. This graph is a tree in which every vertex has the same finite valence, hence the induced map is a surjection. It follows that every 3 -cycle of $\mathscr{F}$ is contained in the image of $\phi$. That is to say, $\phi$ is also a surjection.

It is a well-known fact (indeed, it was known to Dehn [1987, paper 8]) that the automorphisms of $\mathscr{C}(\Sigma)$ are all induced by surface homeomorphisms when $\Sigma$ is either a four-holed sphere or a one-holed torus. This completes the base case of the induction. 
We now furnish the inductive step. Let $\phi: \mathscr{C}(\Sigma) \rightarrow \mathscr{C}(\Sigma)$ be any embedding satisfying the hypotheses of Theorem 1 . Let $\alpha$ be any curve in $\Sigma$. Our previous work on the topological properties of $\phi$ tells us that the complement of $\alpha$ and the complement of $\phi(\alpha)$ are homeomorphic. Therefore, after first composing with a suitable mapping class if need be, $\phi$ restricts to a self-embedding on the curve complex associated to each component of $\Sigma-\alpha$. The embeddings arising in this way are very natural for they inherit many of the properties verified by $\phi$, for instance they also preserve the separating type of a curve. This is of particular relevance when cutting the surface $\Sigma$ along a curve and finding a two-holed torus complementary component. In [Luo 2000], the author explains how to find automorphisms of the curve complex associated to the two-holed torus not induced by a surface homeomorphism. No such automorphism can arise as a restriction, nor can any embedding, as outer curves in this two-holed torus correspond to separating curves in $\Sigma$.

Our inductive hypothesis therefore applies and it tells us that each restriction of $\phi$ associated to a positive complexity component of $\Sigma-\alpha$ is induced by a surface homeomorphism. In gluing back together by identifying the boundary components of $\Sigma-\alpha$ corresponding to $\alpha$, we have a countable family of mapping classes where each such mapping class $f$ satisfies $f(\delta)=\phi(\delta)$ for all $\delta \in X(\alpha) \cup\{\alpha\}$. We must somehow decide which of these, if any, is appropriate.

This construction applies equally well for every curve on $\Sigma$, in particular any curve $\beta$ adjacent to $\alpha$. The set of mapping classes associated to $\alpha$ and the set of mapping classes associated to $\beta$ have nonempty intersection. That is, to the edge of $\mathscr{C}(\Sigma)$ spanned by $\alpha$ and $\beta$, we can associate at least one mapping class $f$ with $f(\delta)=\phi(\delta)$ for all $\delta \in X(\alpha) \cup X(\beta)$.

We need to verify that for any three curves $\alpha, \beta_{1}$ and $\beta_{2}$ such that $\alpha$ is adjacent to both $\beta_{1}$ and $\beta_{2}$, the action on $\mathscr{C}(\Sigma)$ of any such mapping class $f_{1}$ associated to the edge $\alpha, \beta_{1}$ agrees with that of any such mapping class $f_{2}$ associated to the edge $\alpha, \beta_{2}$. For almost all surfaces, $f_{1}$ and $f_{2}$ will be the same mapping class. For now there remains the possibility that $\Sigma-\alpha$ has an exceptional surface component and in particular the possibility that $f_{1}^{-1} f_{2}$ Dehn twists around $\alpha$, or a combination of the two. We treat this in the following lemma.

Lemma 16. Suppose that $\alpha, \beta_{1}, \beta_{2} \in X(\Sigma)$ are distinct, with $\beta_{1}$ and $\beta_{2}$ of zero or otherwise minimal intersection and $\alpha$ disjoint from both $\beta_{1}$ and $\beta_{2}$. Suppose $f_{1}, f_{2} \in$ Map $\Sigma$ are two mapping classes such that $f_{i}(\delta)=\phi(\delta)$ for all $\delta \in X(\alpha) \cup$ $X\left(\beta_{i}\right)$, for $i=1$, 2. Then, $f_{1}^{-1} f_{2} \in \operatorname{Ker} \Sigma$.

Proof. Let $f$ denote the mapping class $f_{1}^{-1} f_{2}$, noting $f$ acts trivially on $X(\alpha)$, and suppose for contradiction that $f \notin \operatorname{Ker} \Sigma$. As we shall see in the subsequent paragraphs, there then exist disjoint (possibly equal) curves $\delta_{1}$ and $\delta_{2}$ on $\Sigma$ 


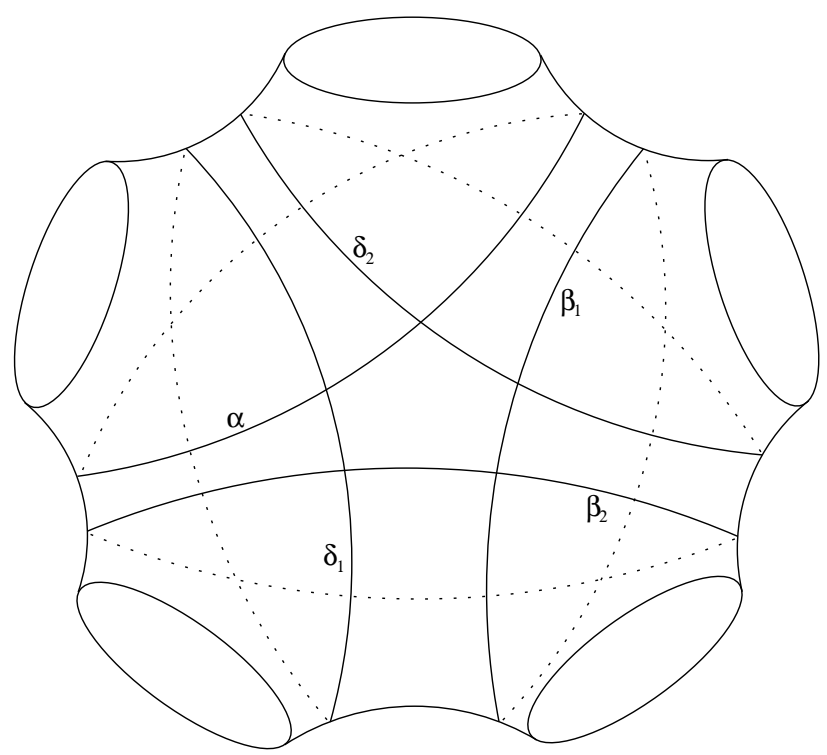

Figure 3. The case of $\Sigma$ a five-holed sphere; $\delta_{1}$ is the only curve on $\Sigma$ disjoint from both $\beta_{1}, \delta_{2} \in X\left(\delta_{1}\right) \cap\left(X(\alpha) \cup X\left(\beta_{2}\right)\right)$. As it happens, $X\left(\delta_{1}\right) \cap\left(X(\alpha) \cup X\left(\beta_{2}\right)\right)$ equals $\left\{\beta_{1}, \delta_{2}\right\}$ in this instance.

such that at least one of $\iota\left(\delta_{i}, \alpha\right)$ and $\iota\left(\delta_{i}, \beta_{i}\right)$ is zero, for both $i=1,2$, and such that $\iota\left(\delta_{1}, f\left(\delta_{2}\right)\right)>0$. Given this, we also have $\iota\left(\delta_{1}, f\left(\delta_{2}\right)\right)=\iota\left(\delta_{1}, f_{1}^{-1} f_{2}\left(\delta_{2}\right)\right)=$ $\iota\left(f_{1}\left(\delta_{1}\right), f_{2}\left(\delta_{2}\right)\right)=\iota\left(\phi\left(\delta_{1}\right), \phi\left(\delta_{2}\right)\right)=0$. This is a contradiction, and we deduce the statement of the lemma.

To see that such a pair of curves $\delta_{1}, \delta_{2}$ must exist, we can argue as follows. Suppose $\delta_{1} \in X\left(\beta_{1}\right)$ has minimal intersection with $\alpha$ and zero or minimal intersection with $\beta_{2}$. Then, $\delta_{1}$ is entirely determined by the nonempty set $X\left(\delta_{1}\right) \cap(X(\alpha) \cup$ $\left.X\left(\beta_{2}\right)\right)$. More precisely, $\delta_{1}$ is the only curve on $\Sigma$ intersecting $\alpha$ and disjoint from every curve in $X\left(\delta_{1}\right) \cap\left(X(\alpha) \cup X\left(\beta_{2}\right)\right)$. See Figure 3 for one example in the fiveholed sphere, in this case a pentagon configuration as described in [Luo 2000].

Suppose for contradiction that $\iota\left(\delta_{1}, f\left(\delta_{2}\right)\right)=0$ for any curve $\delta_{2} \in X\left(\delta_{1}\right) \cap$ $\left(X(\alpha) \cup X\left(\beta_{2}\right)\right)$. Then, $f\left(X\left(\delta_{1}\right) \cap\left(X(\alpha) \cup X\left(\beta_{2}\right)\right)\right) \subseteq X\left(\delta_{1}\right) \cap\left(X(\alpha) \cup X\left(\beta_{2}\right)\right)$. However, because $f$ is a mapping class this inclusion is an equality and we deduce $f\left(\delta_{1}\right)=\delta_{1}$. As the complement in $\Sigma$ of $\beta_{1}$ is filled by a set of curves all fixed by $f$, we deduce $f$ acts trivially on $X\left(\beta_{1}\right)$. Arguing along similar lines, by reinterpreting our contention as $\iota\left(\delta_{2}, f^{-1}\left(\delta_{1}\right)\right)=0$ we deduce $f$ acts trivially on $X\left(\beta_{2}\right)$ as well.

We have shown that $f(\delta)=\delta$ for all $\delta \in X(\alpha) \cup X\left(\beta_{1}\right) \cup X\left(\beta_{2}\right)$. However $X(\alpha) \cup X\left(\beta_{1}\right) \cup X\left(\beta_{2}\right)$ fills $\Sigma$, that is every curve on $\Sigma$ has nonzero intersection with some curve from this set. It follows that $f$ in fact fixes every curve on $\Sigma$. Therefore, $f \in \operatorname{Ker} \Sigma$ and by assumption this is absurd. 
The link of $\alpha$ is either chain-connected, so that for any two of its vertices, $\beta_{1}$ and $\beta_{2}$, there is a sequence of curves $\beta_{1}=\delta_{1}, \delta_{2}, \ldots, \delta_{n}=\beta_{2}$ each distinct and disjoint from $\alpha$ and such that consecutive curves $\delta_{i}, \delta_{i+1}$ have minimal intersection, or is connected. By applying Lemma 16 inductively, we conclude that any two edges ending on $\alpha$ are prescribed the same automorphism of $\mathscr{C}(\Sigma)$ and that any such automorphism is induced by a mapping class. Since $\mathscr{C}(\Sigma)$ is connected, it follows that every edge is allocated the same such automorphism $\Phi$.

All we need do now is verify that this automorphism is equal to $\phi$ everywhere. To do this, we only need to remark that any curve $\alpha$ spans an edge with a second curve $\beta$. This edge is prescribed the automorphism $\Phi$ which, by construction, agrees with $\phi$ on both $X(\alpha)$ and $X(\beta)$. In particular, $\Phi$ agrees with $\phi$ on $X(\beta)$ which contains $\alpha$. This completes one proof of Theorem 1 .

\section{Acknowledgements}

The author thanks the Department of Mathematical and Computing Sciences at the Tokyo Institute of Technology for its warm and generous hospitality, and it is his pleasure to thank Brian Bowditch, Javier Aramayona, Jason Behrstock, Dan Margalit, Graham Niblo and Caroline Series for their enthusiasm and for being so generous with their time; their comments on this work improved the exposition beyond his own capabilities. Last, but by no means least, he wishes to thank the EPSRC for supporting this research.

\section{References}

[Behrstock and Margalit 2006] J. A. Behrstock and D. Margalit, "Curve complexes and finite index subgroups of mapping class groups", Geom. Dedicata 118 (2006), 71-85. MR 2239449 Zbl 05046839

[Bell and Margalit 2005] R. W. Bell and D. Margalit, "Injections of Artin groups", Preprint, 2005. To appear in Comment. Math. Helv. math.GR/0501051

[Bell and Margalit 2006] R. W. Bell and D. Margalit, "Braid groups and the co-Hopfian property", J. Algebra 303:1 (2006), 275-294. MR 2253663 Zbl 05065127

[Birman 1974] J. S. Birman, Braids, links, and mapping class groups, Ann. of Math. Stud. 82, Princeton University Press, Princeton, NJ, 1974. MR 51 \#11477 Zbl 0305.57013

[Brendle and Margalit 2004] T. E. Brendle and D. Margalit, "Commensurations of the Johnson kernel”, Geom. Topol. 8 (2004), 1361-1384. MR 2005j:57041 Zbl 1079.57017

[Brock et al. 2004] J. F. Brock, R. D. Canary, and Y. N. Minsky, "The classification of Kleinian surface groups, II: The ending lamination conjecture”, Preprint, 2004. math.GT/0412006

[Dehn 1987] M. Dehn, Papers on group theory and topology, edited by J. Stillwell, Springer, New York, 1987. MR 88d:01041

[Farb and Ivanov 2005] B. Farb and N. V. Ivanov, "The Torelli geometry and its applications: research announcement”, Math. Res. Lett. 12:2-3 (2005), 293-301. MR 2006c:57001 Zbl 1073.57012 
[Harvey 1981] W. J. Harvey, "Boundary structure of the modular group", pp. 245-251 in Riemann surfaces and related topics (Stony Brook, 1978), edited by I. Kra and B. Maskit, Ann. of Math. Stud. 97, Princeton Univ. Press, Princeton, N.J., 1981. MR 83d:32022 Zbl 0461.30036

[Harvey and Korkmaz 2005] W. J. Harvey and M. Korkmaz, "Homomorphisms from mapping class groups”, Bull. London Math. Soc. 37:2 (2005), 275-284. MR 2005i:57020 Zbl 1066.57020

[Irmak 2004a] E. Irmak, "Complexes of non-separating curves and mapping class groups", preprint, 2004. math.GT/0407285

[Irmak 2004b] E. Irmak, "Superinjective simplicial maps of complexes of curves and injective homomorphisms of subgroups of mapping class groups", Topology 43:3 (2004), 513-541. MR 2005g: $57035 \mathrm{Zbl} 1052.57024$

[Irmak 2006] E. Irmak, "Superinjective simplicial maps of complexes of curves and injective homomorphisms of subgroups of mapping class groups. II", Topology Appl. 153:8 (2006), 1309-1340. MR 2007b:57003 Zbl 1100.57020

[Ivanov 1992] N. V. Ivanov, Subgroups of Teichmüller modular groups, Translations of Mathematical Monographs 115, American Mathematical Society, Providence, RI, 1992. MR 93k:57031 Zbl 0776.57001

[Ivanov 1997] N. V. Ivanov, "Automorphisms of complexes of curves and of Teichmüller spaces", Internat. Math. Res. Notices 1997:14 (1997), 651-666. MR 98j:57023 Zbl 0890.57018

[Ivanov and McCarthy 1999] N. V. Ivanov and J. D. McCarthy, "On injective homomorphisms between Teichmüller modular groups, I", Invent. Math. 135:2 (1999), 425-486. MR 99m:57014 Zbl 0978.57014

[Korkmaz 1999] M. Korkmaz, "Automorphisms of complexes of curves on punctured spheres and on punctured tori”, Topology Appl. 95:2 (1999), 85-111. MR 2000d:57025 Zbl 0926.57012

[Luo 2000] F. Luo, “Automorphisms of the complex of curves”, Topology 39:2 (2000), 283-298. MR 2000j:57045 Zbl 0951.32012

[Margalit 2004] D. Margalit, "Automorphisms of the pants complex”, Duke Math. J. 121:3 (2004), 457-479. MR 2004m:57037 Zbl 1055.57024

[Schmutz Schaller 2000] P. Schmutz Schaller, "Mapping class groups of hyperbolic surfaces and automorphism groups of graphs", Compositio Math. 122:3 (2000), 243-260. MR 2001g:57033 Zbl 0981.57004

[Shackleton 2005] K. J. Shackleton, Aspects of the curve complex and the mapping class group, Ph.D. thesis, University of Southampton, 2005.

[Viro 1972] O. J. Viro, "Links, two-sheeted branching coverings and braids", Mat. Sb. (N.S.) 87:129 (1972), 216-228. MR $45 \# 7701$

Received July 27, 2005.

KENNETH J. SHACKLETON

Department of Mathematical and Computing Sciences

TOKYO INSTITUTE OF TECHNOLOGY

2-12-1 O-OKAYAMA MEgURO-KU

TOKYO 152-8552

JAPAN

shackleton.k.aa@m.titech.ac.jp

kjs2006@alumni.soton.ac.uk 